\title{
Recognition memory for faces: When familiarity supports associative recognition judgments
}

\author{
A. P. YONELINAS, N. E. A. KROLL, I. G. DOBBINS, and M. SOLTANI \\ University of California, Davis, California
}

\begin{abstract}
Recognition memory for single items can be dissociated from recognition memory for the associations between items. For example, recognition tests for single words produce curvilinear receiver operating characteristics (ROCs), but associative recognition tests for word pairs produce linear ROCs. These dissociations are consistent with dual-process theories of recognition and suggest that associative recognition relies on recollection but that item recognition relies on a combination of recollection and assessments of familiarity. In the present study, we examined associative recognition ROCs for facial stimuli by manipulating the central and external features, in order to determine whether linear ROCs would be observed for stimuli other than arbitrary word pairs. When the faces were presented upright, familiarity estimates were significantly above zero, and the associative ROCs were curvilinear, suggesting that familiarity contributed to associative judgments. However, presenting the faces upside down effectively eliminated the contribution of familiarity to associative recognition, and the ROCs were linear. The results suggest that familiarity can support associative recognition judgments, if the associated components are encoded as a coherent gestalt, as in upright faces.
\end{abstract}

The examination of receiver operating characteristics (ROCs) has played an important role in testing theories of human memory (e.g., Ratcliff, Sheu, \& Gronlund, 1992). In a recent study of recognition memory, Yonelinas (1997) found that item recognition tests (e.g., was this word previously presented?) led to curvilinear, asymmetrical ROCs but that associative recognition tests (e.g., were these two words previously paired together?) led to linear ROCs. These results were predicted on the basis of a dual-process model of recognition, in which recognition memory judgments can be based either on the assessment of familiarity, a process that is well described by classical signal-detection theory, or on the basis of a threshold recollection process, whereby subjects retrieve qualitative information about a previous study episode. If performance relies on recollection and familiarity, as is thought to be the case in item recognition, the model predicts that the ROCs should be curvilinear and asymmetrical. In contrast, if performance relies exclusively on recollection (see, e.g., Clark, 1992; Clark \& Gronlund, 1996; Hockley, 1991, 1992; Humphreys, 1978), the model predicts a linear ROC (see Yonelinas, 1994).

The finding that associative ROCs were linear suggests that familiarity does not support associative recognition judgments. However, there may be cases in which familiarity can support such judgments. For example, if

This research was supported by Grants from the National Institutes of Health MS17778 and MH59352-01. Correspondence concerning this article should be addressed to A. P. Yonelinas, Department of Psychology, University of California, Davis, CA 95616 (e-mail: apyonelinas@ucdavis.edu). two items were encoded as a coherent whole (or gestalt), familiarity might be useful in discriminating between studied and rearranged pairs of items. That is, if the two items were encoded as a coherent whole, that whole item, as well as its constituent parts, might increase in familiarity when it was studied. In this way, a repeated pair might be more familiar than a rearranged pair.

To test this hypothesis, we examined associative memory for integrated facial stimuli. Internal facial features (e.g., mouth, nose, and eyes) were paired with external facial features (e.g., hair, chin, and shoulders), as in Figure 1 , and we tested subjects' ability to discriminate repeated faces from rearranged faces (i.e., internal features of one studied face presented with the external features of another studied face). The reason for examining associative recognition with parts of faces was that, unlike the randomly selected word pairs that had been previously examined, each face formed a well-integrated whole.

Previous behavioral and neuropsychological data suggest that faces are encoded holistically rather than as separable components (for reviews, see Moscovitch, Winocur, \& Behrmann, 1997; Searcy \& Bartlett, 1996). If correct, this means that repeated faces may be more familiar than their individual features, so that later associative judgments should benefit from assessments of familiarity, leading the associative ROCs to become curvilinear.

Faces are particularly useful in the present context because it is possible to control whether faces are treated as coherent wholes or as a conjunction of separate features by presenting faces either upright or upside down. Studies examining the effects of inverting faces have suggested that when faces are presented upright, they are en- 
coded holistically, but when they are inverted, they are treated as a conjunction of separate features (for a review, see Searcy \& Bartlett, 1996). For example, a subject's ability to make judgments about the relationship between features within a face is impaired when faces are presented upside down, but the ability to make judgments about the individual features is not affected by inversion (see, e.g., Bartlett \& Searcy, 1993; Searcy \& Bartlett, 1996). Thus, when a face is studied and tested in an upright orientation, it should be encoded as a coherent whole, rather than as two separate items. This, in turn, should allow familiarity to support associative judgments, and consequently, the ROC should become curvilinear. However, when faces are inverted, they should be encoded as conjunctions of separate parts. Familiarity should then not support associative judgments, and the resulting ROC should be linear.

In the present experiment, recognition ROCs were examined under three different conditions. Subjects were presented with drawings of faces and were instructed to try to remember each face. They were then given a recognition test in which they rated the confidence of their memory responses. In the upright condition, faces were presented in an upright orientation at study and test, and the subjects were required to discriminate between repeated faces and rearranged faces. In the upside down condition, the task was identical, except that the faces were presented upside down at study and at test. In the control condition, faces were studied and tested in an upright orientation, but the test required the subjects to discriminate between repeated faces and completely novel faces. The latter condition was used in order to determine whether the item recognition ROC for the facial stimuli used in the present study was similar to those reported in previous recognition memory studies for words.

The contribution of recollection and familiarity was estimated by fitting a dual-process model to the observed ROCs and by examining the linearity of the ROCs. The estimation method, which is described in the Method section, has been found to provide estimates of recollection and familiarity that parallel those provided by Jacoby's (1991) process dissociation procedure and Tulving's (1985) remember-know procedure (Yonelinas, Dobbins, Szymanski, Dhaliwal, \& King, 1996; Yonelinas \& Jacoby, 1995). By estimating the contribution of recollection and familiarity, it will be possible to determine whether familiarity contributes to performance in the associative tests. If familiarity contributes to performance, the parameter estimate for familiarity should be greater than zero. If the faces in the upside down condition are not treated as whole items, familiarity should not contribute to associative performance, and familiarity should be close to zero. In contrast, if the faces are treated as whole items when they are presented upright, familiarity should contribute to performance, and the estimate of familiarity should be greater than zero. The contribution of familiarity was also assessed by conducting a linearity analysis on the observed ROCs. If familiarity does not contribute to performance, the ROC should be linear, and if it does contribute, the ROC should be curvilinear.

\section{METHOD}

\section{Subjects}

Sixty-six undergraduate students from the University of California at Davis participated in the experiment and received extra credit in an introductory psychology course. Fourteen subjects were randomly assigned to each of the three test conditions (upright, upside down, and control). For reasons discussed below, an additional 14 subjects were tested in the upside down condition.

\section{Materials, Design, and Procedure}

Colored faces of men, women, and children (see Figure 1) were constructed so that the external features (i.e., hair, head shape, ears, and visible clothing) and the central features (i.e., eyes, eyebrows, nose, mouth, and facial markings) could be switched. The faces were approximately 4-6 in. wide and 5-7 in. high when presented on the 15-in. computer monitor.

The subjects first studied 120 faces and were warned that, in the later memory test, the distractors might be composed of parts of the studied faces. Consequently, they were instructed to pay careful attention to the relationships between the components of each face. The subjects viewed each item twice-first, at a rate of one face every $4 \mathrm{sec}$, and second, after being informed that they were now going to see the same faces for a second study trial, at a rate of $2 \mathrm{sec}$ per face.

The test phase began immediately after the study phase. The subjects made memory judgments, using a 6-point confidence rating scale that ranged from certain it is new (1) to certain it is old (6). The test phase was subject paced, with the subjects indicating their answers by pressing the appropriate numerical key on the computer keyboard. Each subject was presented with 120 test faces (60 repeats and 60 distractors) in a random order. In the upright condition, the repeats were in the study list, and the distractors consisted of rearranged faces from the study list (i.e., the internal features had been studied with another set of external features, and the external features had been studied with another set of internal features). The faces were constructed so that, across subject, each whole face (internal and external features) served equally often as a repeated and as a rearranged face. In the upside down condition, the design was identical, except that the faces were presented upside down during study and test. In the control condition, the distractor faces were completely new faces that had not been presented in the study list. In the test phase, the subjects were informed about the design of the target and the lure items that they would receive.

\section{RESULTS}

The ROCs were plotted as a function of response confidence and were fit to a dual-process model, to derive estimates of recollection and familiarity. Justification and empirical support for the model assumptions will be described in the Discussion section. The model assumes that an old item will be accepted as old if it is recollected $(R)$ or if it is not recollected $(1-R)$ and if its familiarity exceeds the response criterion. Familiarity is assumed to reflect a Gaussian equal-variance signal-detection process (see Macmillan \& Creelman, 1991). The probability of accepting an item on the basis of familiarity is rep- 


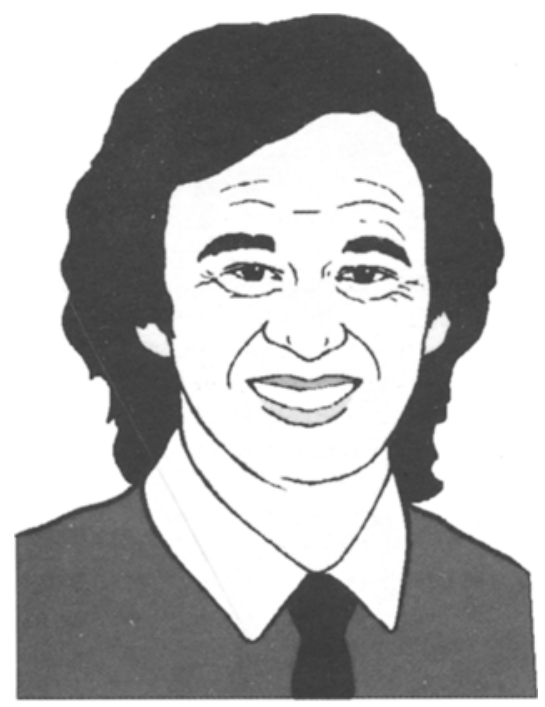

Study

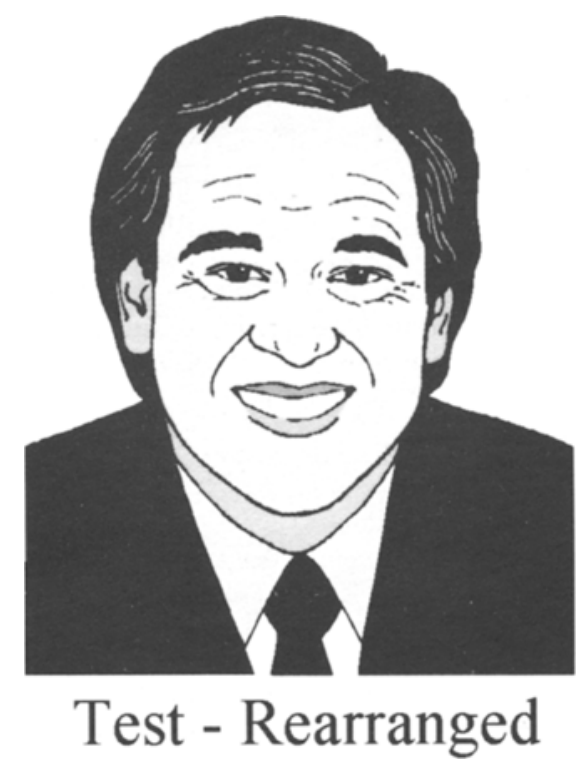

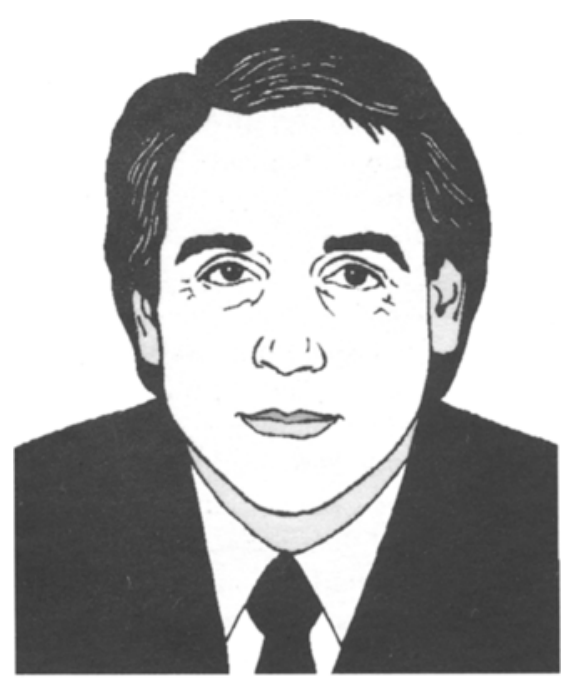

Study

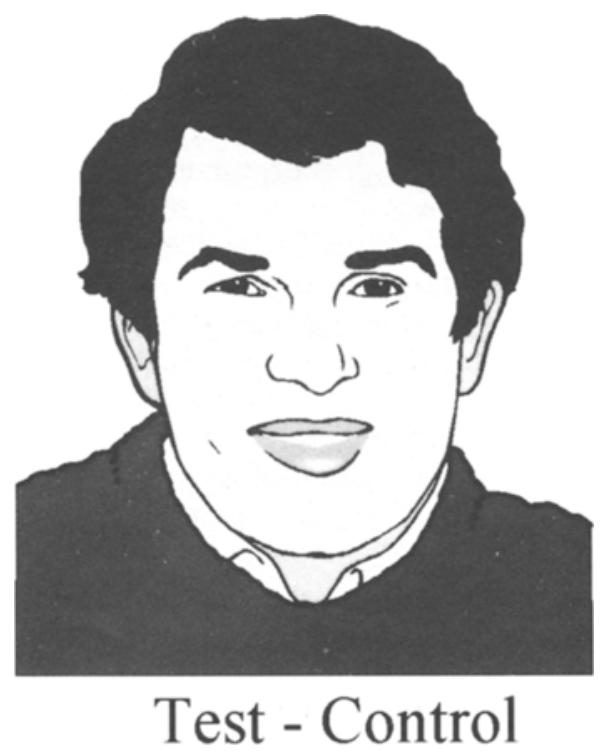

Figure 1. Examples of facial stimuli used in the study phase (top panels). In the associative test, the distractors were recombinations of studied faces ( $\mathrm{e}$.g, the bottom left panel). In the item test, the distractors were new to the experiment (e.g., the bottom right panel).

resented as $\Phi\left(d^{\prime} / 2-c_{i}\right)$, which represents the proportion of the old item familiarity distribution that exceeds the response criterion $\left(c_{i}\right)$, given that the distance between the old and the new distributions is $d^{\prime}$. The probability of accepting a new item will be equal to the probability that its familiarity exceeds the response criterion $\left[\Phi\left(-d^{\prime} / 2-\right.\right.$ $\left.c_{i}\right)$ ]. Thus, the hit rate and the false alarm rate can be represented as follows:

$$
P(\text { "yes" } \mid \text { old })_{i}=R+(1-R) \Phi\left(d^{\prime} / 2-c_{i}\right),
$$

$$
P(\text { "yes" } \mid \text { new })_{i}=\Phi\left(-d^{\prime} / 2-c_{i}\right) \text {. }
$$

These two equations represent performance at one point on the ROC. ' An ROC with five points requires 10 equations. Assuming that memory ( $R$ and $d^{\prime}$ ) remains constant across the ROC and only the response criterion $\left(c_{i}\right)$ varies, the set of equations can be solved to derive estimates of $R$ and $d^{\prime}$. A search algorithm was used to find the best-fitting parameters for these equations by reducing the sum of squared errors between the predicted and the 


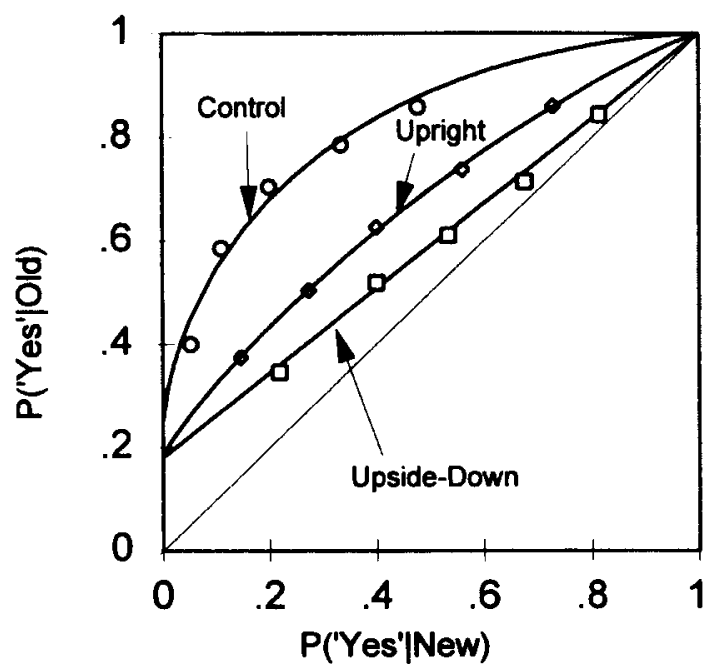

Figure 2. The average receiver operating characteristics for the upright, upside down, and control conditions, fit to the dualprocess model.

observed ROC points (see Yonelinas, Kroll, Dobbins, Lazzara, \& Knight, 1998). A log-likelihood estimation method was also used, and it produced very similar parameter estimates.

The average ROCs are presented in Figure 2, along with the functions derived by fitting the dual-process model to each ROC. An examination of Figure 2 shows that the model provided an accurate account of the ROC data in each condition. An examination of the statistical fit of the model to the average ROCs showed that it accounted for more than $99.3 \%$ of the variance in each condition.

The parameter estimates are presented in Table 1. The parameter estimates based on the average ROCs were similar to the average parameter estimates derived when each subject's ROC was fit to the model, suggesting that the average ROCs were representative of the individual ROCs. An examination of the parameter estimates showed that familiarity contributed to associative recognition judgments when the faces were presented in an upright orientation, but that familiarity did not support performance when the faces were presented upside down. That is, estimates of familiarity were significantly greater than zero in the upright condition $[t(13)=2.12]$ but were not sig- nificantly greater then zero in the upside down condition $[t(13)=0.40]$.

To further quantify the ROCs, a linearity analysis was conducted, in which the average ROCs were first fit to a linear function, then to a quadratic function. Note that, because the $x$ - and $y$-axes are arbitrary, and the fit of the quadratic will vary, depending on which axis is regressed onto the other, the quadratic was fit in both directions, and the best of these two fits was used to measure the contribution of the nonlinear component. The average control ROC exhibited a significant linear component $[F(1,3)=$ $\left.19.19, M S_{\mathrm{e}}=0.005\right]$, but it was fit significantly better by a quadratic function $\left[F(1,2)=72.70, M S_{\mathrm{e}}=0.004\right]$, indicating that the function was curvilinear. The ROC in the upside down condition was fit well by a linear function $\left[F(1,3)=981.19, M S_{\mathrm{e}}=0.0002\right]$, and the fit was not improved when the quadratic component was introduced $(F<1)$, showing that the function was linear. The average ROC in the upright condition was fit reasonably well by a linear function $\left[F(1,3)=246.38, M S_{\mathrm{e}}=0.001\right]$, but it was fit significantly better by a quadratic function $[F(1,2)=$ $42.56, M S_{\mathrm{e}}=0.001$ ], showing that the ROC was curvilinear. Thus, the linearity analyses supported the conclusions drawn from the dual-process model: Familiarity did not contribute to associative judgments when the faces were upside down (i.e., the ROC was linear), but it did contribute when the faces were upright (i.e., the ROC was curvilinear).

Were the low levels of performance in the upside down condition responsible for the low familiarity estimates in that condition? As recognition performance decreases, the ROC will approach the diagonal. Thus, the ROC will tend to become more linear, and this will produce lower estimates of familiarity. However, inverting the faces led to a significant decrease in familiarity $[t(13)=2.05]$ but did not influence recollection $[t(13)=0.50]$, suggesting that, in general, inverting the faces did not make the task more difficult; rather, it selectively reduced the utility of familiarity as a basis for associative judgments.

In any case, in order to determine whether familiarity would contribute to performance in the upside down condition when overall performance was increased, an additional 14 subjects were tested in the upside down condition, and the subjects were then divided into two groups. The 14 subjects with the highest hit-minus-false-alarm

Table 1

Estimates (E) of Recollection $(R)$ and Familiarity $\left(d^{\prime}\right)$, Derived From the Average Receiver Operating Characteristics (ROCs) in

Each Test Condition, and the Average Parameter Estimates (PE), Derived When the Model Was Fit to Each Subject's ROC

\begin{tabular}{|c|c|c|c|c|c|c|c|c|}
\hline \multirow[b]{3}{*}{ Parameter } & \multicolumn{8}{|c|}{ Condition } \\
\hline & \multicolumn{2}{|c|}{ Control } & \multicolumn{2}{|c|}{ Upright } & \multicolumn{2}{|c|}{ Upside Down } & \multicolumn{2}{|c|}{ High Upside Dowr } \\
\hline & $\mathrm{E}$ & $\mathrm{PE}$ & $\mathrm{E}$ & PE & $\mathrm{E}$ & $\mathrm{PE}$ & $\mathrm{E}$ & PE \\
\hline$R$ & .24 & .18 & .18 & .21 & .18 & .18 & .27 & .28 \\
\hline$d^{\prime}$ & 1.04 & 1.15 & 0.34 & 0.26 & -0.02 & -0.01 & 0.04 & 0.01 \\
\hline
\end{tabular}




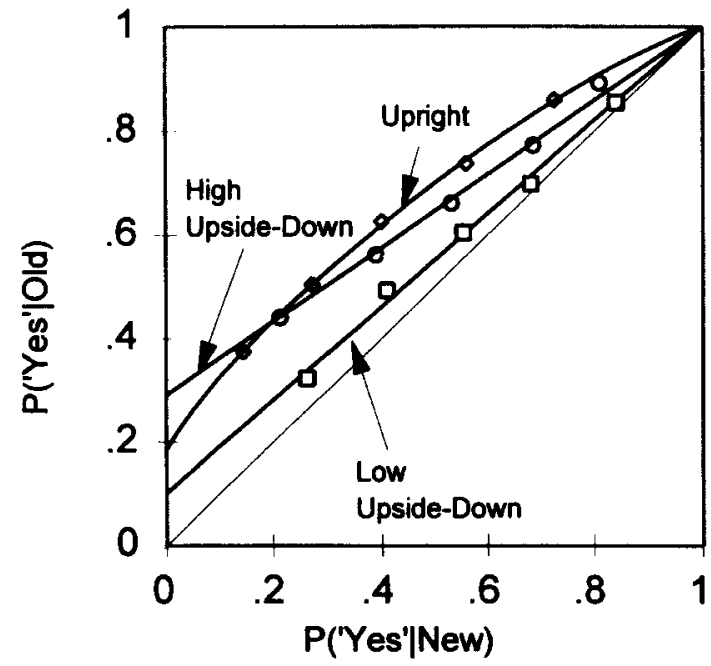

Figure 3. The average receiver operating characteristics for the high upside down group, the low upside down group, and the upright group, fit to the dual-process model.

score at the highest level of response confidence were included in the high group, and the other 14 subjects were included in the low group.

The ROCs for the high and the low upside down groups are presented in Figure 2, along with the ROC from the upright condition. An examination of Figure 2 shows that the ROC for the upside down condition was still linear even for the high group. A linearity analysis was conducted on the average ROC of the high group, and it showed that the function exhibited a significant linear component $\left[F(1,3)=712.13, M S_{\mathrm{e}}=0.0003\right]$ and that introducing a quadratic did not improve the fit over that of a linear model $\left[F(1,2)=11.125, M S_{\mathrm{e}}=0.001\right]$, indicating that the ROC was not curved. Importantly, the ROC for this group was close to that of the upright group - in fact, the two functions intersect at the left side of the ROC. Thus, even when the two groups perform approximately equally well, the ROC in the upright condition was curvilinear, and the ROC in the upside down condition was linear.

Most important, the familiarity estimates for the high upside down group (see Table 1) did not differ significantly from zero $[t(13)=0.19]$. These results are consistent with the previous analysis in showing that familiarity does not appear to contribute to the associative memory judgments when the faces are presented upside down.

There are two potential problems with the preceding linearity analyses. First, the regression analysis assumes that the data points are independent. Because the ROCs are cumulative functions, this assumption is not met. Second, the standard linearity analysis uses a specific curvilinear function, the quadratic, to fit the data, and there may be other curvilinear functions (e.g., exponential) that fit the data better. To address these concerns, we performed a second set of analyses based on the raw fre- quency data. If the cumulative function is linear with a constant slope, for any given change in the false alarm rate, there will be a proportional change in the hit rate. In other words, the odds of success for each of the confidence levels (excluding level 6 , which serves as the intercept) should be constant. If, however, the cumulative function is curvilinear, there will be a change in the odds of success across confidence levels. An examination of the response frequencies showed that there was no effect of response confidence on the odds of success for the original upside down condition $\left[\chi^{2}(4)=4.63\right]$ or for the high upside down group $\left[\chi^{2}(4)=7.50\right]$, showing that the ROCs were linear. In contrast, the odds of success varied significantly across response confidence for the upright $\left[\chi^{2}(4)=15.14\right]$ and control $\left[\chi^{2}(4)=190.98\right]$ conditions, showing that these ROCs were not linear. Thus, an examination of the frequency counts led to the same conclusions as did the standard linearity analysis reported earlier.

To facilitate comparison of the results with those of previous studies, the ROCs were plotted on $z$-coordinates. A linear function was fit to each function, to determine the slope and intercept, and then a quadratic was introduced, to determine whether the ROCs were significantly curvilinear. Note that a linear ROC should exhibit a slight Ushape in $z$ space. For the control condition, the slope and intercept values were .82 and 1.15 respectively, and the function was not significantly curvilinear $[F(1,2)=12.63$, $\left.M S_{\mathrm{e}}=0.004\right]$. For the upright condition, the slope and intercept values of the line of best fit for the $z$-ROC were .80 and 0.51 , respectively, and the function was not significantly curvilinear $\left[F(1,2)=8.81, M S_{\mathrm{e}}=0.001\right]$. For the initial upside down condition, the slope and intercept values were .83 and 0.24 , respectively. The function did exhibit a slight $\mathrm{U}$-shape, but it was not significantly curved $\left[F(1,2)=1.37, M S_{\mathrm{e}}=0.003\right]$. However, for the high upside down condition, the slope and intercept values were .81 and 0.42 , and the function was significantly $U$-shaped $\left[F(1,2)=921.2, M S_{\mathrm{e}}=0.001\right]$.

\section{DISCUSSION}

When faces were presented in an upright orientation in an associative recognition test, the estimates of familiarity were greater than zero, and the ROC was curvilinear, suggesting that familiarity was used to discriminate between repeated and rearranged faces. In contrast, when the faces were presented upside down, the estimates of familiarity were not greater than zero, and the ROC was linear, suggesting that familiarity was not used to discriminate between repeated and rearranged faces in this condition. This dissociation between the upright and the upside down conditions was also observed when the overall levels of performance were controlled.

The linear associative ROC observed when the faces were upside down replicates previous findings with associative recognition for word pairs (Yonelinas, 1997). 
However, the curvilinear ROC observed for the upright faces shows that curvilinear associative ROCs can be observed under conditions in which familiarity was expected to discriminate between repeated and rearranged items.

The ROCs were fit quite well by the dual-process signaldetection model (see Figures 2 and 3). An alternative model, the unequal-variance signal-detection model (see Macmillan \& Creelman, 1991), can account for only some of the ROCs. If the unequal-variance model is correct, the ROCs must be linear when plotted in $z$-space. The $z$-ROCs for the control and upright conditions were fit quite well by linear functions. However, the $z$-ROCs for the upside down conditions were $U$-shaped, indicating that the Gaussian assumption underlying that model was violated.

The results of the present experiment suggest that the distinction between recollection and familiarity is not isomorphic with the distinction between associative and item information (cf. Hockley, 1992; Yonelinas, 1997). Although it may be the case that associative judgments generally do not benefit from assessments of familiarity, the present results show that there are cases in which familiarity can support such discriminations. These results suggest that if performance on an associative memory test is used as an index of recollection, one should be careful to ensure that the paired items are not treated as coherent wholes. The present results support the notion that faces can be treated as items when they are presented in an upright orientation, but that when they are presented upside down, they are treated as separate sets of features (cf. Searcy \& Bartlett, 1996).

These results may provide insights into the current debate about the ability of amnesics to learn new associations. Several studies have indicated that amnesics exhibit a disproportionate deficit in recollection and that their recognition memory judgments rely primarily on familiarity (e.g., Huppert \& Piercy, 1976; Yonelinas et al., 1998). Moreover, early reports found that amnesics were unable to learn new associations (e.g., Shimamura \& Squire, 1989), suggesting that recollection may be necessary in order to form novel associations. However, more recent studies of humans (e.g., Keane, Gabrieli, Nolan, \& McNealy, 1995; Moscovitch, Winocur, \& McLachlan, 1986), rats, and nonhuman primates (e.g., Eichenbaum, Otto, \& Cohen, 1994) show that, under some conditions, amnesics can acquire novel associations. This indicates that recollection may not be necessary in order to form novel associations. The present results suggest that earlier studies may have failed to obtain associative learning in amnesics because the associated items were encoded as separate components, rather than holistically.

The present results are consistent with two different models of familiarity: an activation view and an episodic binding view. First, studying a face in an upright orientation may increase the activation of preexisting representations in memory that are similar to that face (e.g., a face may be similar to someone you know), and this increase in activation may be used as a basis for accurate associative judgments. Activation of representations for single components of a face would not be expected to support associative judgments, but it would support item judgments. Inverted faces are not similar to existing facial representations, so they should not greatly influence the activation of existing representations, and familiarity should not support associative judgments. A second possibility is that familiarity may be capable of binding separate features together to create new episodic representations. Thus, as with current global memory models (see Clark \& Gronlund, 1996), new items and new associations are encoded into memory and can serve as a basis for familiaritybased recognition judgments.

Although the results of the linearity analyses converge with the conclusions drawn from the estimates derived with the dual-process model, there are a number of critical assumptions that underlie the model, and it is useful to consider the evidence supporting those assumptions. First, the model assumes that recollection and familiarity are independent. The evidence for this assumption, derived from behavioral studies of healthy humans, has been discussed previously (for a review, see Jacoby, Yonelinas, \& Jennings, 1997), and those points will not be reiterated here. Note, however, that to the extent that the model accurately accounted for the present ROC data, these results provide indirect support for that underlying model assumption. Additional support for the independence assumption comes from event-related potential studies that demonstrate that recollection and familiarity are associated with independent electrophysiological correlates (Düzel, Yonelinas, Mangun, Heinze, \& Tulving, 1997). Further support for the independence assumption comes from single-cell recording studies and from lesion studies of rats and nonhuman primates that show that two regions in the medial temporal lobe (i.e., the hippocampal and parahippocampal regions) subserve two distinct recognition memory retrieval functions (for reviews, see Aggleton \& Brown, in press; Eichenbaum et al., 1994).

A second critical assumption is that familiarity reflects a classical signal-detection process. Most important is that the familiarity distributions are assumed to be normally distributed and old and new item distributions are assumed to have equal variance. We have tested these assumptions by examining recognition memory ROCs of amnesics patients (Yonelinas et al., 1998). Because these patients have severe deficits in recollection, their ROCs should provide an index of familiarity. Unlike the recognition ROCs of healthy subjects, the amnesics' ROCs were curvilinear and symmetrical, as would be expected if familiarity reflected an equal-variance signal-detection process. Also note that the equal-variance assumption is consistent with some computational models, such as TODAM (Murdock, 1982).

A third critical assumption is that recollection reflects a threshold retrieval process. What this means is that either subjects will be successful at retrieving information 
about a prior event or they will fail. Subjects may retrieve many different things about a prior event, only some of which may be useful in a specific test context; however, there will be cases in which they are unable to retrieve any information that supports accurate discriminations. The finding that the ROCs for the upside down faces were linear provides direct support for this assumption. Moreover, the linear ROCs in that condition suggest that, when subjects recollected information about an item, this led to a high-confidence recognition response and that the responses associated with lower levels of response confidence did not reflect any additional memory sensitivity.

One potential limitation to the dual-process model is that it does not explicitly represent false recollections. The model only measures correct recollection: the ability to correctly discriminate between target and lure faces. However, subjects may sometimes falsely recollect faces that were not studied, and this may influence the shape of the observed ROC. False recollection would not greatly alter the shape of the linear ROCs, but it could alter the shape of the curvilinear ROCs. For the linear ROCs, if the subject falsely recollected items, this would increase the false alarm rate and the hit rate by some constant proportion. This would have the effect of shifting the ROC points toward the 1,1 intercept. However, it would not influence the shape of the function, because it intersects the 1,1 intercept. For the curvilinear ROCs, false recollection would also increase the hit and false alarm rates toward the 1,1 intercept, but because the function is curvilinear, it essentially pinches the end points of the ROC together, forcing the function to exhibit an exaggerated inverted U-shape. We have tested several different ways of incorporating false recollection into the model equations, and ROC pinching appeared in each case. Furthermore, an informal examination of several recognition ROCs suggested that the data do sometimes deviate from the model in a manner consistent with the notion that false recollection is occurring. For example, a careful examination of the control ROC in Figure 2 shows that the ROC is slightly more curvilinear than the dual-process model predicts. Whether false recollection plays a significant role in recognition ROCs is not yet clear, but future studies examining the possibility will be informative.

In conclusion, the present study demonstrates that linear and curvilinear associative ROCs can be observed in tests of associative recognition. As with previous experiments using random word pairs, associative judgments for upside down faces led to linear ROCs. However, when faces were presented upright, the associative ROC was curvilinear. Estimates of recollection and familiarity showed that familiarity contributed to associative recognition memory judgments when the faces were upright but did not do so when the faces were upside down. The results suggest that when separate items are treated holistically, assessments of familiarity can support associative recognition memory judgments.

\section{REFERENCES}

Aggleton, J. P., \& Brown, M. W. (in press). Episodic memory, amnesia and the hippocampal-anterior thalamic axis. Behavioral \& Brain Sciences.

Bartlett, J. C., \& SearCy, J. H. (1993). Inversion and configuration of faces. Cognitive Psychology, 25, 281-316.

CLARK, S. E. (1992). Word frequency effects in associative and item recognition. Memory \& Cognition, 20, 231-243.

Clark, S. E., \& Gronlund, S. D. (1996). Global matching models of recognition memory: How the models match the data. Psychonomic Bulletin \& Review, 3, 37-60.

Düzel, E., Yonelinas, A. P., Mangun, G. R., Heinze, H., \& Tulving, E. (1997). Event-related brain potential correlates of two states of conscious awareness in memory. Proceedings of the National Academy of Sciences, 94, 5973-5978

Eichenbaum, H., Otto, T., \& Cohen, N. J. (1994). Two functional components of the hippocampal memory system. Behavioral \& Brain Sciences, 17, 449-518.

HoCKLEY, W. E. (1991). Recognition memory for item and associative information: A comparison of forgetting rates. In W. E. Hockley \& S. Lewandowsky (Eds.), Relating theory and data: Essays on human memory in honor of Bennet B. Murdock (pp. 227-248). Hillsdale, NJ: Erlbaum.

HOCKLEY, W. E. (1992). Item versus associative information: Further comparisons of forgetting rates. Journal of Experimental Psychology: Learning, Memory, \& Cognition, 18, $1321-1330$.

HumphrEYs, M. S. (1978). Item and relational information: A case for context independent retrieval. Journal of Verbal Learning \& Verbal Behavior, 17, 175-187.

Huppert, F. A., \& Piercy, M. (1976). Recognition memory in amnesic patients: Effects of temporal context and familiarity of material. Cortex, 12, 3-20

JACOBY, L. L. (1991). A process dissociation framework: Separating automatic from intentional uses of memory. Journal of Memory \& Language, 30, 513-541.

Jacoby, L. L., Yonelinas, A. P., \& Jennings, J. M. (1997). The relationship between conscious and unconscious (automatic) influences: A declaration of independence. In J. Cohen \& J. W. Schooler (Eds.), Scientific approaches to the question of consciousness (pp. 13-47). Hillsdale, NJ: Erlbaum.

Keane, M. M., Gabrieli, J. D. E., Nolan, J. S., \& McNealy, S. I. (1995). Normal perceptual priming of illegal nonwords in amnesia. Journal of the International Neuropsychological Society, 1, 124-133.

Macmillan, N. A., \& Creelman, C. D. (1991). Detection theorv: A user's guide. Cambridge: Cambridge University Press.

Moscovitch, M., Winocur, G., \& Behrmann, M. (1997). What is special about face recognition? Nineteen experiments on a person with visual object agnosia and dyslexia but normal face recognition. Journal of Cognitive Neuroscience, 9, 555-604.

Murdock, B. B. (1982). A theory of storage and retrieval of item and associative information. Psychological Review, 89, 609-666.

RatclifF, R., Sheu, C. F., \& Gronlund, S. D. (1992). Testing global memory models using ROC curves. Psychological Review, 3, 518535.

Searcy, J. H., \& Bartlett, J. C. (1996). Inversion and processing of component and spatial-relation information in faces. Journal of Experimental Psychology: Human Perception \& Performance, 22, 904915.

Shimamura, A. P., \& Squire, L. R. (1989). Impaired priming of new associations in amnesia. Journal of Experimental Psychology: Learning, Memory, \& Cognition, 15, 721-728.

Tulving, E. (1985). Memory and consciousness. Canadian Psychologist, 26, $1-12$.

Yonelinas, A. P. (1994). Receiver-operating characteristics in recognition memory: Evidence for a dual-process model. Journal of Experimental Psychology: Learning, Memory, \& Cognition, 20, 1341 1354 
YONFLINAS, A. P. (1997). Recognition memory ROCs for item and associative information: The contribution of recollection and familiarity. Memory \& Cognition, 25, 747-763.

Yonelinas, A. P., Dobins, I., Szymanski, M. D., Dhaliwal, H. S., \& KING, L. (1996). Signal detection, threshold, and dual-process models of recognition memory: ROCs and conscious recollection. Consciousness \& Cognition, 5, 418-441.

Yonelinas, A. P., \& Jacoby, L. L. (1995). The relation between remembering and knowing as bases for recognition: Effects of size congruency. Journal of Memory \& Language, 34, 622-643.

Yonelinas, A. P., Kroll, N. E. A., Dobbins, I. G., Lazzara, M., \& KNIGHT, R. T. (1998). Recollection and familiarity deficits in amnesia: Convergence of remember-know, process dissociation, and receiver operating characteristic data. Neuropsychology, 12, 1-17.

\section{NOTE}

1. Note that subjects may also be able to recollect that new items are new, and thus, an additional $R$ parameter may be needed. However, in order to simplify the model, we assumed that subjects would not often recollect a new item as new. The accurate fit provided by the model suggests that this simplifying assumption was reasonable under these test conditions.

(Manuscript received June 3, 1998; revision accepted for publication February 17, 1999.) 